\title{
How to Manipulate Through-Space Conjugation and Clusterolumi- nescence of Simple AIEgens with Isolated Phenyl Rings?
}

\author{
Jianyu Zhang, ${ }^{\dagger}$ Lianrui Hu, ${ }^{\dagger}$ Kaihua Zhang, ${ }^{\ddagger}$ Junkai Liu, ${ }^{\dagger}$ Xingguang Li, ${ }^{\dagger}$ Haoran Wang, ${ }^{\dagger}$ Zhaoyu \\ Wang, ${ }^{\dagger}$ Herman H. Y. Sung, ${ }^{\dagger}$ Ian D. Williams, ${ }^{\dagger}$ Zebing Zeng, ${ }^{\ddagger}$ Jacky W. Y. Lam, ${ }^{\dagger}$ Haoke Zhang, ${ }^{*},{ }^{\perp}$ and \\ Ben Zhong Tang*,+,\|,\#
}

†Department of Chemistry, Hong Kong Branch of Chinese National Engineering Research Center for Tissue
Restoration and Reconstruction and Institute for Advanced Study, The Hong Kong University of Science and
Technology, Clear Water Bay, Kowloon, Hong Kong, China

§MOE Key Laboratory of Macromolecular Synthesis and Functionalization, Department of Polymer Science and Engineering, Zhejiang University, Hangzhou 310027, China

₹State Key Laboratory of Chemo/Biosensing and Chemometrics, College of Chemistry and Chemical Engineering, Hunan University, Changsha 410082, China

${ }^{\perp}$ Guangdong Provincial Key Laboratory of Luminescence from Molecular Aggregates, South China University of Technology, Guangzhou 510640, China

"Shenzhen Institute of Aggregate Science and Technology, School of Science and Engineering, The Chinese University of Hong Kong, Shenzhen 518172, China

\#AIE Institute, Guangzhou Development District, Guangzhou 510530, China

\begin{abstract}
Apart from the traditional through-bond conjugation (TBC), through-space conjugation (TSC) is gradually proved as another important interaction in photophysical processes, especially for the recent observation of clusteroluminescence from nonconjugated molecules. However, unlike TBC in conjugated chromophores, it is still challenging to manipulate TSC and clusteroluminescence. Herein, simple and nonconjugated triphenylmethane (TPM) and its derivatives with electron-donating and electron-withdrawing groups were synthesized, and their photophysical properties were systematically studied. TPM was characterized with visible clusteroluminescence due to the intramolecular TSC. Experimental and theoretical results showed that the introduction of electron-donating groups into TPM could red-shift the wavelength and increase the efficiency of clusteroluminescence simultaneously, due to the increased electronic density and stabilization of TSC. However, TPM derivatives with electron-withdrawing groups showed inefficient or even quenched clusteroluminescence caused by the vigorous excited-state intramolecular motion and intermolecular photoinduced electron transfer process. This work provides a reliable strategy to manipulate TSC and clusteroluminescence.
\end{abstract}

\section{INTRODUCTION}

The use of light is the starting point for human civilization, which plays a vital role in the development of our society. Beginning from 5th B.C. when philosophers of ancient Greece raised the question about the essence of light, humanity started to investigate and utilize natural sources of light, such as sun and firelight. ${ }^{1}$ In modern society, because of the deeper cognition on science and technology, people began to design artificial emitters, especially luminogens which can emit efficiently upon photoexcitation. ${ }^{2-5}$ Therefore, what are the laws behind the luminescent behaviors and how to design and manipulate artificial luminophores at different levels of hierarchical structures are important issues for scientists, as evidenced by the Nobel Prize in Chemistry in 2008 for the discovery of green fluorescent protein, and Physics in 2014 for the invention of efficient blue light-emitting diodes, respectively.

Compared with inorganic materials, organic fluorophores show better flexibility, biocompatibility, processability, structural diversity, etc. Thus, they show great promise in practical application. Researchers have established many photophysical theories based on molecular science, which focus on the fluorescent mechanisms of organic fluorophores. For example, efficient fluorophores are closely correlated with large $\pi$ conjugation through covalent bonds (e.g. double bond, triple bond or aromatic ring), ${ }^{6-7}$ and introduction of donor (D) and acceptor (A) groups into the molecular skeleton often leads to redder but weaker emission, ${ }^{8-9}$ especially for molecules showing twisted intramolecular charge transfer effect. ${ }^{10-11}$ In most cases, these molecular theories are capable of revealing the photophysical mechanisms of organic luminescent materials. Based on these studies at molecular level, a paradigm of material research is gradually formed that molecular structures and properties always determine the performance of macroscopic materials. However, due to the continuous development of photophysical research, some abnormal phenomena were reported sporadically that aggregates performed entirely different properties 
from single-molecule species. One example is the aggregation-caused quenching (ACQ) effect where most fluorophores are highly emissive in solution but become non-emissive in the aggregate state. ${ }^{12}$ In contrast, some invisible phenomena of molecules in the isolated state could be observed in the aggregate state, ${ }^{13-15}$ such as the aggregation-induced emission (AIE) effect. Luminogens with AIE effect (AIEgens) are non-emissive in dilute solution but luminesce strongly in the aggregate state. The flourishing development of ACQ and AIE-related studies brings a paradigm shift from molecular science to aggregate science. ${ }^{16-18}$

Recent research in aggregate science has reported that many nonconjugated structures, such as polystyrene, ${ }^{19}$ peptide, ${ }^{20}$ and maleimide, ${ }^{21}$ are non-emissive in solution but can emit visible light in the solid state. This unconventional photophysical process is termed as clusterization-triggered emission and the corresponding emission is known as clusteroluminescence. ${ }^{22-25}$ The previous studies suggested that strong intermolecular interactions among the closely packed molecules generated new emissive species and stabilized excited excitons to produce clusteroluminescence. ${ }^{26-28}$ Apart from intermolecular interaction, intramolecular through-space conjugation (TSC) was also proved to play an important role in clusteroluminescence. For example, our recent study revealed that nonconjugated 1,1,2,2-tetraphenylethane with weak intermolecular interactions fluoresced efficiently in the aggregate state, and the intramolecular TSC among the four phenyl rings contributed greatly to the clusteroluminescence. ${ }^{29}$ Based on the above results, two common questions are emerged: i) is TSC a general mechanism for clusteroluminescence, and ii) is it possible to manipulate TSC in clusteroluminogens?

In this work, nonconjugated triphenylmethane (TPM) and its derivatives with different electron-donating and withdrawing groups, namely 4,4',4"trimethoxytriphenylmethane (TPM-MO), 4,4',4"'-tris $(N, N$ dimethylaminophenyl)methane (TPM-DMA), 4,4',4"tricyanotriphenylmethane (TPM-CN) and 4,4',4"trinitrotriphenylmethane $\left(\mathrm{TPM}-\mathrm{NO}_{2}\right)$, were synthesized and satisfactorily characterized (Figures S1-S11 in Supporting Information). All the molecules showed visible light in the solid state with different colors and efficiency. Experimental and theoretical results proved that TSC was also applicable to these clusteroluminogens. The electrondonating groups in TPM-MO and TPM-DMA could red-shift the wavelength and increase efficiency of clusteroluminescence simultaneously by enhancing the TSC. However, the electron-withdrawing groups in TPM-CN and TPM-NO $\mathrm{N}_{2}$ decentralized the electrons of phenyl rings and destabilized the intramolecular TSC, resulting in low emission efficiency. On the other hand, the restriction of intramolecular motions (RIM), ${ }^{30}$ which served as the mechanism of AIE phenomenon, helped to stabilize the preferential conformation for TSC in the aggregate state. The synergistic effect between TSC and RIM generated efficient clusteroluminescence of TPM and its derivatives. Thus, this study not only verified the general mechanism of TSC in clusteroluminogens but also provided a reliable strategy to manipulate clusteroluminescence at the molecular level.

\section{Results and Discussion}

2.1 Photophysical properties. TPM is a simple molecule with three isolated phenyl rings and was first prepared to study its photophysical properties. The absorption spectra of TPM both in THF solution and solid state exhibited an absorption maximum $\left(\lambda_{\mathrm{ab}}\right)$ corresponding to benzene ring at $264 \mathrm{~nm}$, confirming the nonconjugated nature of the molecule (Figure 1a). In pure THF solution, TPM showed one emission peak at $285 \mathrm{~nm}$ which was attributed to the intrinsic through-bond conjugation (TBC) of benzene. Addition of poor solvent such as water into the THF solution induced the formation of TPM aggregates (Figure 1b). During the aggregation process, the emission intensity at $285 \mathrm{~nm}$ first increased and then decreased, and the turning point was located at a water fraction $\left(f_{\mathrm{w}}\right)$ of $50 \%$ in volume. It was noteworthy that a new broad emission peak appeared at 350-380 $\mathrm{nm}$ at $f_{\mathrm{w}}=80-90 \%$, and its intensity at $400 \mathrm{~nm}$ was 17 times higher than that in pure THF solution (Figure 1c). This long-wavelength emission demonstrated the AIE phenomenon and might be attributed to the formation of stable through-space interaction in the aggregate state that gave rise to clusteroluminescence. ${ }^{29} \mathrm{At}$ $f_{\mathrm{w}}$ of less than $50 \%$, the enhancement of TBC emission was due to the increased polarity of the solvent mixture. ${ }^{31}$ However, aggregates merely formed at high water fraction whose internal polarity was less affected by the surrounding environment. Meanwhile, a portion of the TBC emission was reabsorbed by the TSC species via the Förster resonance energy transfer (FRET) process, which decreased the emission from TBC but increased the emission from TSC. The solid-state emission spectrum of TPM showed two maximums $\left(\lambda_{\mathrm{em}}\right)$ at 288 and $402 \mathrm{~nm}$ under $270 \mathrm{~nm}$ excitation (Figure 1d), which were assigned to the intrinsic emission from TBC of phenyl rings and clusteroluminescence with TSC, respectively. The excitation spectrum of TPM in the solid state exhibited a longwavelength peak at $310 \mathrm{~nm}$, suggesting the formation of new emissive species by TSC. Thus, only one peak with a fluorescence quantum yield ( $\Phi_{\mathrm{TSC}}$ ) of $2.7 \%$ appeared at 403 $\mathrm{nm}$ with $310 \mathrm{~nm}$ excitation (Figure S12). 

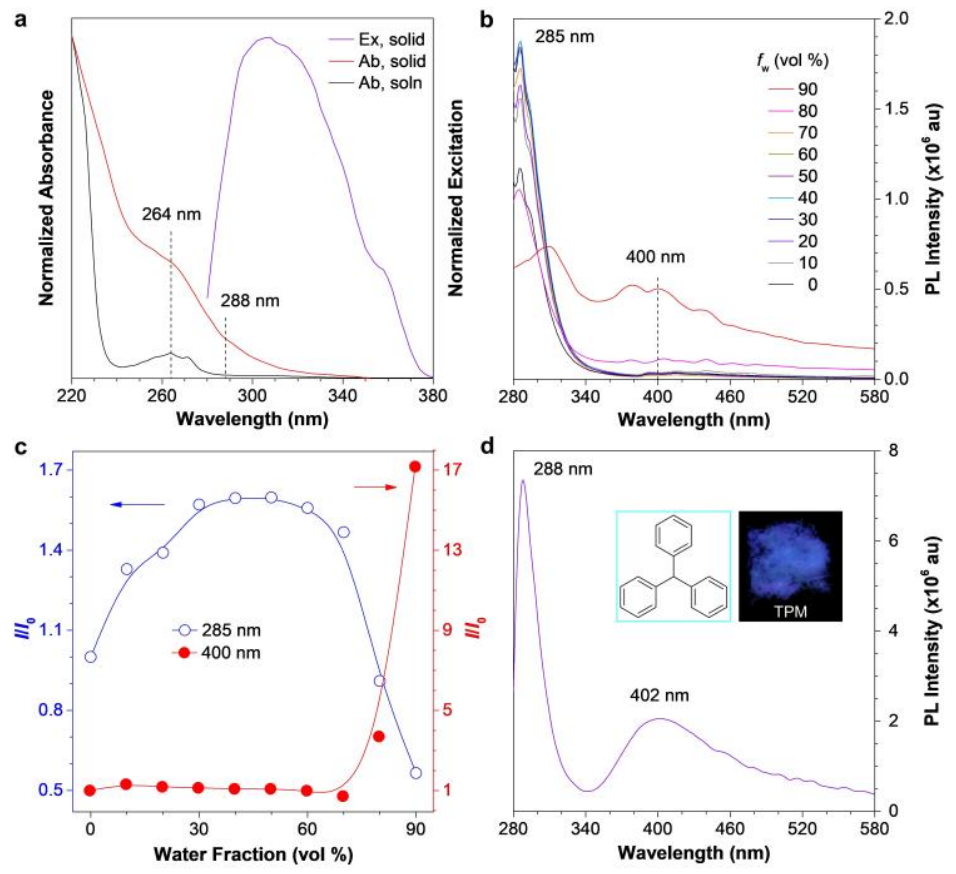

Figure 1. (a) Absorption (Ab) spectra of TPM in THF solution (soln) and solid state. Inset: excitation (Ex) spectrum in the solid state taken at $\lambda_{\mathrm{em}}=402 \mathrm{~nm}$. (b) Photoluminescence (PL) spectra of TPM in THF/water mixtures with different water fractions $\left(f_{\mathrm{w}}\right)$. (c) Plots of relative PL intensity $\left(I / I_{0}\right)$ versus $f_{\mathrm{w}}$ at different emission wavelengths. Concentration $=10^{-4} \mathrm{M}, \lambda_{\mathrm{ex}}=270 \mathrm{~nm}, I_{0}=$ intensity at $f_{\mathrm{w}}=0 \%$. (d) PL spectra of TPM in the solid state taken at $\lambda_{\mathrm{ex}}=270 \mathrm{~nm}$. Inset: structure and fluorescent photo of TPM taken under illumination of a $365 \mathrm{~nm}$ UV lamp.
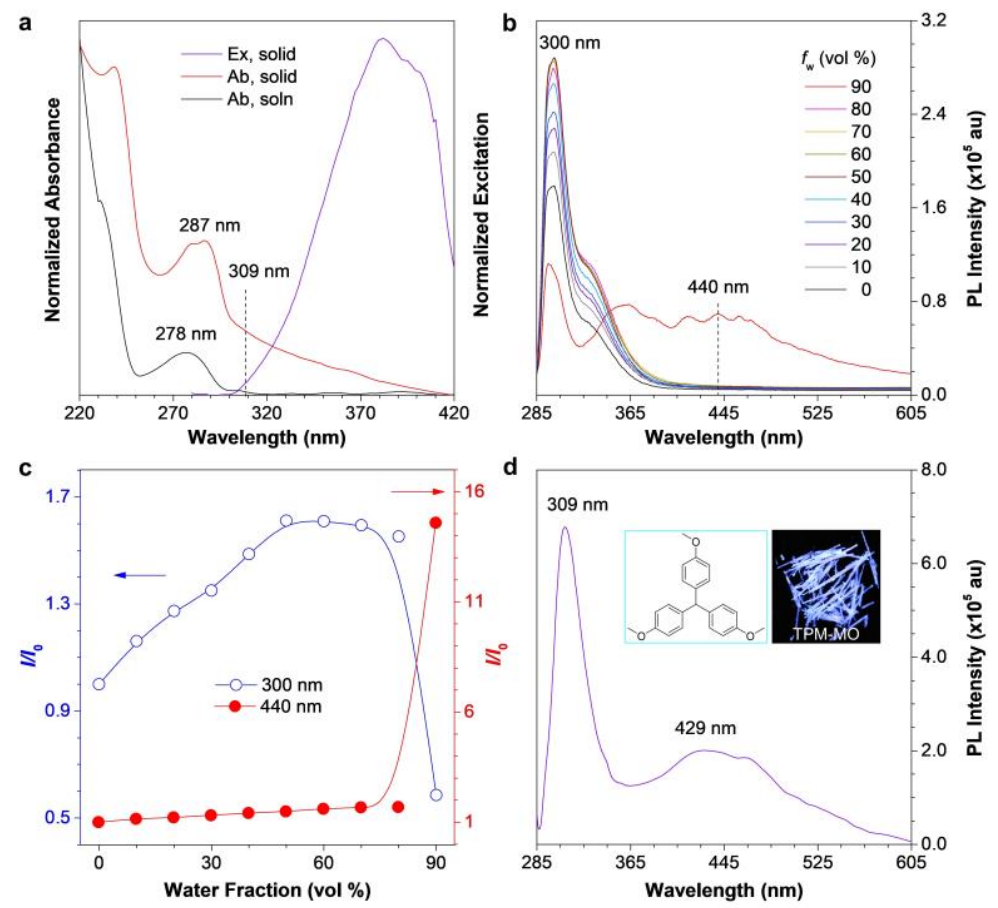

Figure 2. (a) Absorption (Ab) spectra of TPM-MO in THF solution (soln) and solid state. Inset: excitation (Ex) spectrum in the solid state taken at $\lambda_{\mathrm{em}}=429 \mathrm{~nm}$. (b) Photoluminescence (PL) spectra of TPM-MO in THF/water mixtures with different water fractions $\left(f_{\mathrm{w}}\right)$. (c) Plots of relative PL intensity $\left(I / I_{0}\right)$ versus $f_{\mathrm{w}}$ at different emission wavelengths. Concentration $=10^{-4} \mathrm{M}, \lambda_{\mathrm{ex}}=280 \mathrm{~nm}, I_{0}=$ intensity at $f_{\mathrm{w}}=0 \%$. (d) PL spectra of TPM-MO in the solid state taken at $\lambda_{\mathrm{ex}}=280 \mathrm{~nm}$. Inset: structure and fluorescent photo of TPM-MO taken under illumination of a $365 \mathrm{~nm}$ UV lamp. 

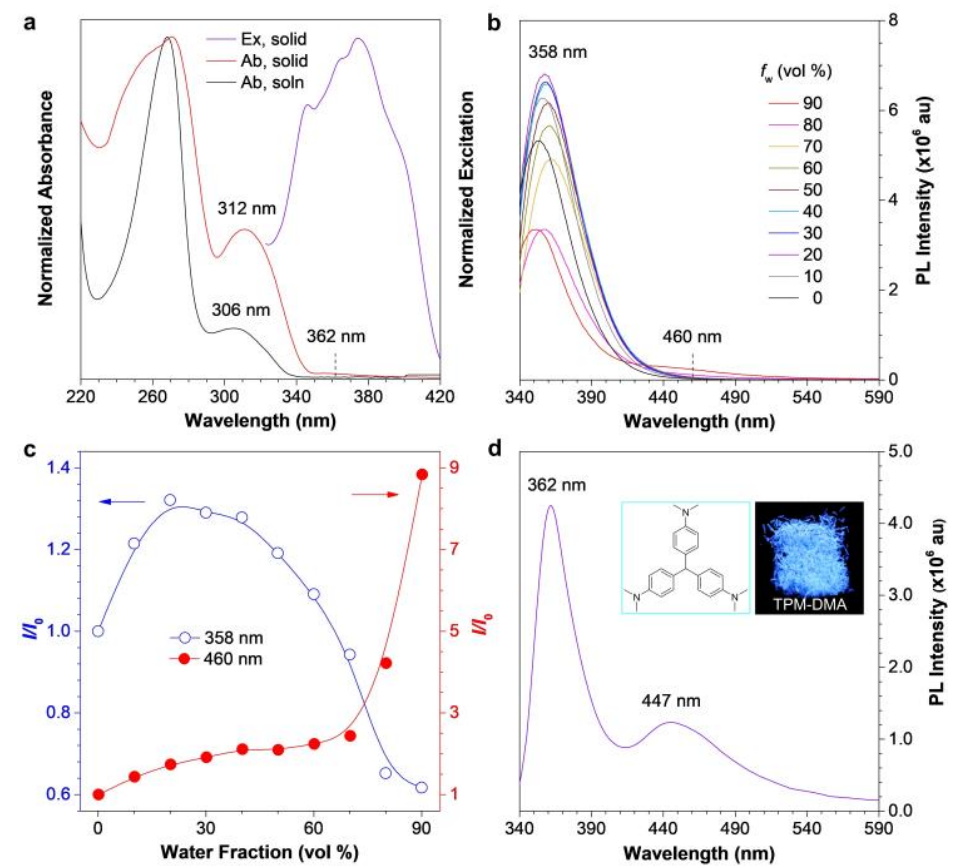

Figure 3. (a) Absorption (Ab) spectra of TPM-DMA in THF solution (soln) and solid state. Inset: excitation (Ex) spectrum in the solid state taken at $\lambda_{\mathrm{em}}=447 \mathrm{~nm}$. (b) Photoluminescence (PL) spectra of TPM-DMA in THF/water mixtures with different water fractions $\left(f_{\mathrm{w}}\right)$. (c) Plots of relative PL intensity $\left(I / I_{0}\right)$ versus $f_{\mathrm{w}}$ at different emission wavelengths. Concentration $=10^{-4} \mathrm{M}, \lambda_{\mathrm{ex}}=320$ $\mathrm{nm}, I_{0}=$ intensity at $f_{\mathrm{w}}=0 \%$. (d) PL spectra of TPM-DMA in the solid state taken at $\lambda_{\mathrm{ex}}=320 \mathrm{~nm}$. Inset: structure and fluorescent photo of TPM-DMA taken under illumination of a $365 \mathrm{~nm}$ UV lamp.

The above results proved the existence of clusteroluminescence in TPM but with a low $\Phi$. To adjust the through-space interaction among the three phenyl rings, electron-donating methoxy groups were introduced to increase the electron density of each phenyl ring. The obtained TPM-MO showed an $\lambda_{\mathrm{ab}}$ at $278 \mathrm{~nm}$ in THF solution (Figure 2a), which was similar to that of anisole (Figure S13). The $\lambda_{a b}$ shifted bathochromically to $287 \mathrm{~nm}$ in the solid state. TPM-MO emitted short-wavelength emission at $300 \mathrm{~nm}$ in THF/water mixture, and its intensity also first increased but later decreased by increasing $f_{\mathrm{w}}$ (Figure $\mathbf{2 b}$ and 2c). At the same time, a new broad peak at $\sim 440 \mathrm{~nm}$ was observed at $f_{\mathrm{w}}=90 \%$, whose intensity was 15 times higher than that in pure THF solution. Also, the emission spectrum was well extended to $600 \mathrm{~nm}$. The solid-state emission spectrum of TPM-MO exhibited two emission peaks at 309 and $429 \mathrm{~nm}$ at $\lambda_{\mathrm{ex}}=280 \mathrm{~nm}$, which originated from TBC and TSC, respectively (Figure 2d). The excitation spectrum of TPM-MO also showed a broad peak at around $370 \mathrm{~nm}$ in the solid state. Upon excitation at $370 \mathrm{~nm}$, the clusteroluminescence of TPM-MO was red-shifted to 439 $\mathrm{nm}$ and the $\Phi_{\mathrm{TSC}}$ was enhanced to $6.3 \%$ in comparison to $\mathrm{TPM}$ with $\lambda_{\mathrm{em}}=403 \mathrm{~nm}$ and $\Phi_{\mathrm{TSC}}=2.7 \%$, respectively (Figure S12).

A strong electron-donating group, $\mathrm{N}, \mathrm{N}$-dimethylamino (DMA) group, was also utilized to construct TPM-DMA. Its $\lambda_{\mathrm{ab}}$ was located at $306 \mathrm{~nm}$ and $312 \mathrm{~nm}$ in pure THF solution and solid state (Figure 3a), respectively, due to the better conjugation and donor (D)-acceptor (A) effect of DMA and phenyl ring. Figure $\mathbf{3 b}$ showed that only one emission peak was detected at $358 \mathrm{~nm}$ at low $f_{\mathrm{w}}$, which was associated with the TBC of $N, N$-dimethylaniline (Figure S13). Similarly, TBC emission became weaker at $f_{\mathrm{w}}$ of more than $50 \%$ (Figure 3c). A long-wavelength emission at $460 \mathrm{~nm}$ was observed at $f_{\mathrm{w}} \geqslant 80 \%$, and its intensity at $f_{\mathrm{w}}=90 \%$ was 9 -fold higher than that in pure THF solution. In the solid state, TPM-DMA exhibited brightly sky-blue clusteroluminescence at $\lambda_{\mathrm{em}}=$ $447 \mathrm{~nm}$ and another invisible TBC emission at $362 \mathrm{~nm}$ with $\lambda_{\mathrm{ex}}=320 \mathrm{~nm}$ (Figure 3d). When excited at the excitation maximum of $380 \mathrm{~nm}$ (Figure 3a), only the long-wavelength emission stemmed from TSC was observed at $453 \mathrm{~nm}$ (Figure S12). The corresponding $\Phi_{\mathrm{TSC}}$ was $6.9 \%$, which slightly higher than that of TPM-MO (6.3\%).

Considering that electron-donating moieties could bathochromic-shift and enhance the $\Phi$ of clusteroluminescence, the effect of electron-withdrawing group on TSC was further explored. Thus, cyano and nitro groups were selected as substituents to synthesize TPM-CN and TPM-NO $\mathrm{N}_{2}$, respectively. The $\lambda_{\mathrm{ab}}$ of TPM-CN in THF solution and solid state was redder than TPM and was both located at $282 \mathrm{~nm}$ (Figure 4a), corresponding to the TBC emission of benzonitrile (Figure S13). As shown in Figure 4b, only the emission of TBC $\left(\lambda_{\mathrm{em}}=294 \mathrm{~nm}\right)$ was observed at low $f_{\mathrm{w}}$ and another long-wavelength peak located at 457 nm was noticed at $f_{\mathrm{w}}=90 \%$ (Figure 4c). In the solid state, two emission peaks appeare d t 300 and $460 \mathrm{~nm}$ at $270 \mathrm{~nm}$ excitation (Figure 4d). The inset in Figure 4a showed that the $\lambda_{\text {ex }}$ was much longer than the $\lambda_{\mathrm{ab}}$ in the solid state. Therefore, upon excitation at $380 \mathrm{~nm}$, only one peak at 482 nm was detected, which was even redder than that of TPMDMA $\left(\lambda_{\mathrm{em}}=453 \mathrm{~nm}\right)$. The $\Phi_{\mathrm{TSC}}$ of such TSC emission was low and was measured to be $2.2 \%$ at $\lambda_{\mathrm{ex}}=380 \mathrm{~nm}$, which was lower than that of TPM, TPM-MO and TPM-DMA. For TPM$\mathrm{NO}_{2}$, its absorption maximum was redder than TPM, but its emission was quenched in both solution and solid state at room temperature and could not be identified (Figure S14). 

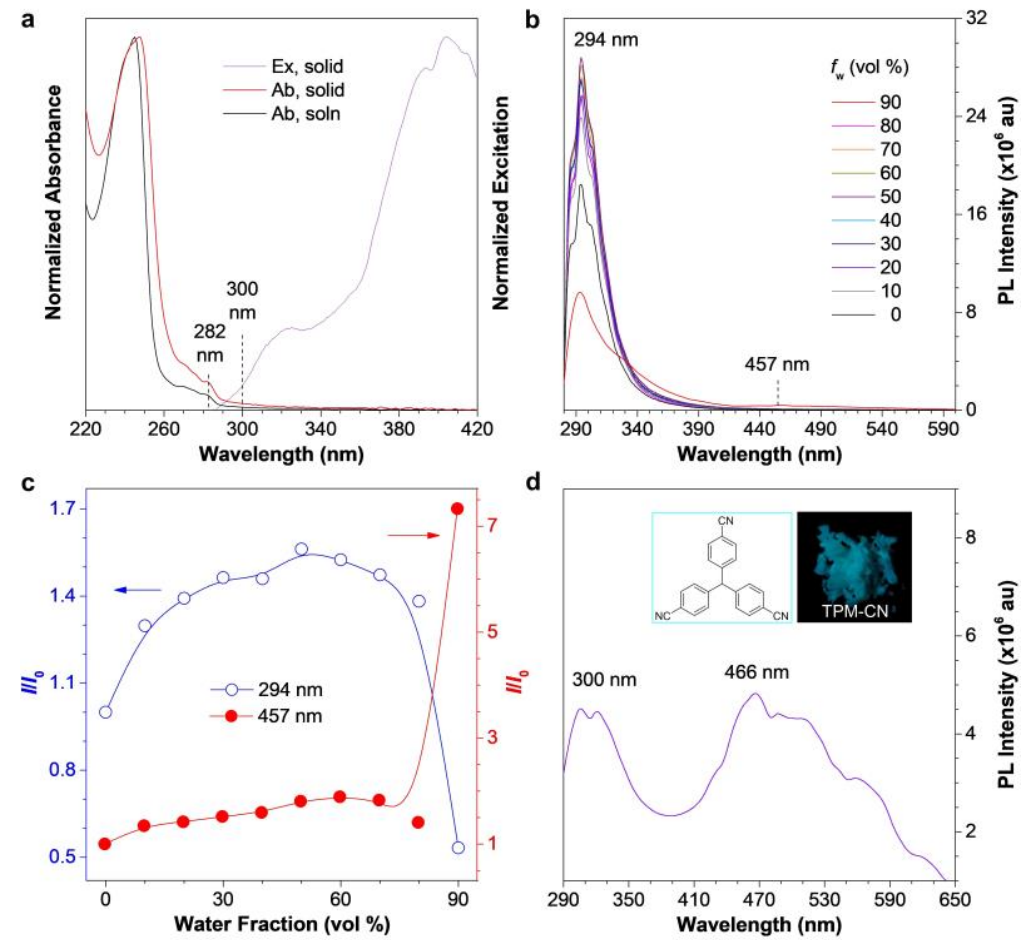

Figure 4. (a) Absorption (Ab) spectra of TPM-CN in THF solution (soln) and solid state. Inset: excitation (Ex) spectrum in the solid state taken at $\lambda_{\mathrm{em}}=466 \mathrm{~nm}$. (b) Photoluminescence (PL) spectra of TPM-CN in THF/water mixtures with different water fractions $\left(f_{\mathrm{w}}\right)$. (c) Plots of relative PL intensity $\left(I / I_{0}\right)$ versus $f_{\mathrm{w}}$ at different emission wavelengths. Concentration $=10^{-4} \mathrm{M}, \lambda_{\mathrm{ex}}=270 \mathrm{~nm}, I_{0}=$ intensity at $f_{\mathrm{w}}=0 \%$. (d) PL spectra of TPM-CN in the solid state taken at $\lambda_{\mathrm{ex}}=270$. Inset: structure and fluorescent photo of TPMCN taken under illumination of a $365 \mathrm{~nm}$ UV lamp.

Table 1. Photophysical Properties of TPM and Its Derivatives (TPM-R) ${ }^{a}$

\begin{tabular}{|c|c|c|c|c|c|c|c|}
\hline \multirow{2}{*}{ TPM-R } & \multirow{2}{*}{$\lambda_{\mathrm{ab}}(\mathrm{nm})$} & \multirow{2}{*}{$\lambda_{\mathrm{ex}, \text { solid }}(\mathrm{nm})$} & \multicolumn{2}{|c|}{ TBC Emission } & \multicolumn{2}{|c|}{ TSC Emission } & \multirow{2}{*}{$\Phi_{\text {TSC }}(\%)$} \\
\hline & & & $\lambda_{\mathrm{TBC}}(\mathrm{nm})$ & $\tau_{\mathrm{TBC}}(\mathrm{ns})$ & $\lambda_{\mathrm{TSC}}(\mathrm{nm})$ & $\tau_{\mathrm{TSC}}(\mathrm{ns})$ & \\
\hline TPM-DMA & 306 & 375 & 362 & 3.29 & 447 & 9.69 & 6.9 \\
\hline TPM-MO & 278 & 382 & 309 & 2.61 & 429 & 3.57 & 6.3 \\
\hline ТPM & 264 & 307 & 288 & $<1$ & 402 & 3.64 & 2.7 \\
\hline TPM-CN & 282 & 404 & 305 & $<1$ & 466 & 2.56 & 2.2 \\
\hline TPM-NO 2 & 276 & & & & & & 0.4 \\
\hline
\end{tabular}

a Abbreviation: $\lambda_{\mathrm{ab}}=$ absorption maximum in THF solution, $\lambda_{\text {ex,solid }}=$ excitation maximum in the solid state measured at emission maximum of $447 \mathrm{~nm}$ (TPM-DMA), $429 \mathrm{~nm}$ (TPM-MO), $402 \mathrm{~nm}$ (TPM) and $466 \mathrm{~nm}(\mathrm{TPM}-\mathrm{CN})$. TBC = through-bond conjugation, $\lambda_{\text {твс }}$ $=$ emission maximum through $\mathrm{TBC}$ in the solid state, $\tau_{\mathrm{TBC}}=$ emission lifetime through $\mathrm{TBC}$ in the solid state, TSC $=$ through-space conjugation, $\lambda_{\mathrm{TSC}}=$ emission maximum through TSC in the solid state, $\tau_{\mathrm{TSC}}=$ emission lifetime through TSC in the solid state, $\Phi_{\mathrm{TSC}}=$ absolute luminescence quantum yield at excitation wavelength of $380 \mathrm{~nm}$ (TPM-DMA), $370 \mathrm{~nm}$ (TPM-MO), $310 \mathrm{~nm}$ (TPM), or 380 $\mathrm{nm}(\mathrm{TPM}-\mathrm{CN})$.

To exclude the interference from radical species which was reported to be responsible for some extrinsic emissions, ${ }^{32-33}$ electron spin resonance spectra of the present molecules were measured, but none of them showed obvious signals in the presence or absence of UV illumination (Figure S15). This result suggested the inherent emission of these stable structures. The photophysical properties of all molecules are summarized in Table 1. It was noteworthy that the emission lifetime caused by TSC ( $\left.\tau_{\mathrm{TSC}}\right)$ was longer than that due to TBC $\left(\tau_{\mathrm{TBC}}\right)$, demonstrating the relatively stable excitons formed by through-space interaction (Figure S16). For example, the $\tau_{\text {TSC }}$ of TPM-DMA was measured to be $9.69 \mathrm{~ns}$, which was three times longer than $\tau_{\mathrm{TBC}}(3.29 \mathrm{~ns})$. Besides, when the substituents were changed from electron-donating to electron-withdrawing groups, the $\tau_{\text {TSC }}$ became shorter and the $\Phi_{\mathrm{TSC}}$ decreased from $6.9 \%$ in TPM-DMA to $0.4 \%$ in TPM$\mathrm{NO}_{2}$. 


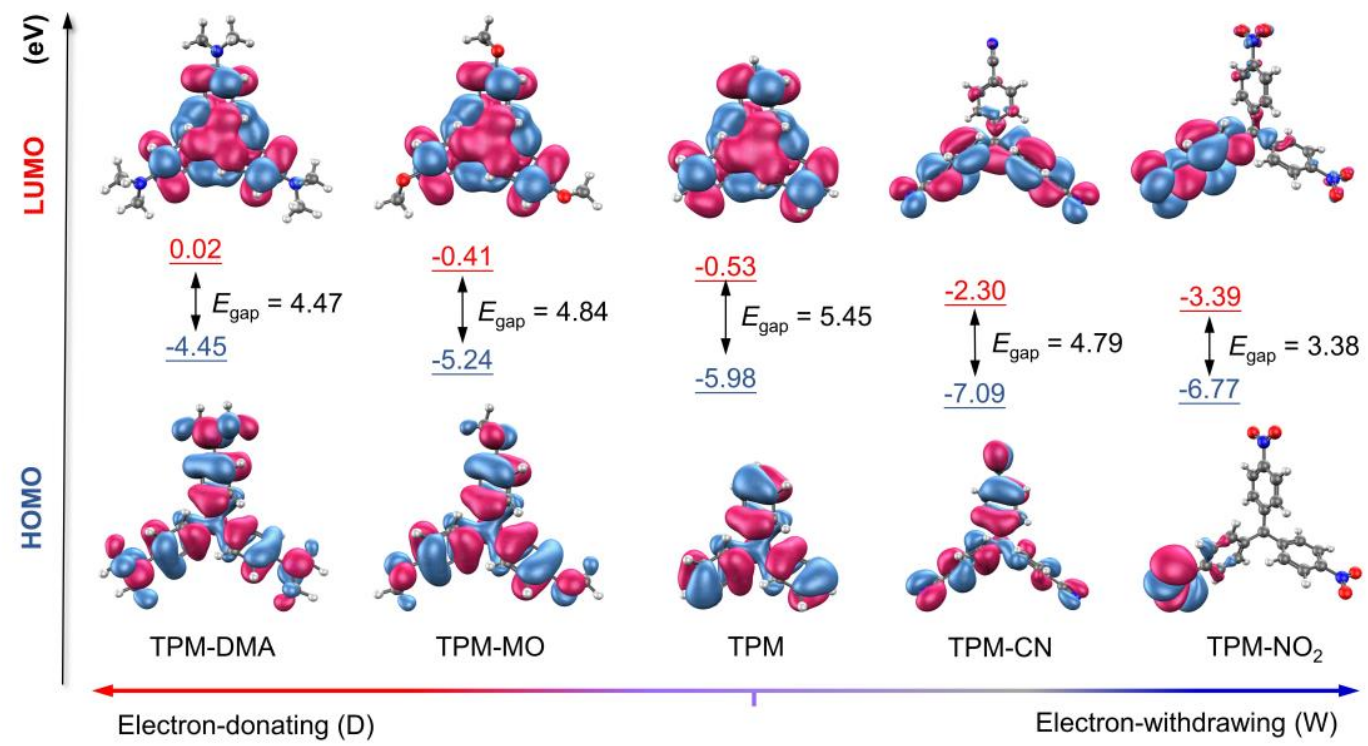

Figure 5. Frontier molecular orbitals of optimized excited-state geometries of TPM derivatives calculated by TD-DFT method at B3LYP-D3/6-31G(d,p) level, Gaussian 16 program.

2.2 Through-space Conjugation. To confirm TSC and investigate the electronic effect on clusteroluminescence, single-molecule calculation on present compounds were carried out. The density functional theory method at B3LYP-D3/6-31G(d,p) level was utilized to calculate the ground- and excited-state geometries. The reference plane was constructed to illustrate the structural information, which was set as atoms of 1-2-3 in Table S1. At ground state $\left(\mathrm{S}_{0}\right)$, all the optimized structures displayed a highly $C_{3}$ symmetrical and propeller-like conformation, as evidenced by the dihedral angles between the reference plane and each of phenyl ring were around $55^{\circ}$. Frontier molecular orbitals at $\mathrm{S}_{0}$ were shown in Figure $\mathbf{S 1 7}$, including electron cloud distribution and energy levels of the highest occupied molecular orbital (HOMO) and the lowest unoccupied molecular orbital (LUMO). Electrons at HOMO were distributed on the phenyl rings and substituent groups.

However, the geometries and electronic effect became different at the excited state $\left(\mathrm{S}_{1}\right)$ (Table $\left.\mathbf{S 1}\right)$. The energy gap between HOMO and LUMO of TPM derivatives were narrower than TPM (Figure 5), which was consistent with their redder $\lambda_{\mathrm{ab}}$ and $\lambda_{\mathrm{em}}$ through TBC. Besides, the values were smaller than the corresponding substituted phenyl rings such as benzene, $\mathrm{N}, \mathrm{N}$-dimethylaniline, anisole, benzonitrile and nitrobenzene, suggesting the existence of intramolecular through-space interaction (Table S2). As observed from the LUMO of TPM, a clear electronic overlapping among the three isolated phenyl rings was observed, which verified the effect of intramolecular TSC in TPM. For TPM-MO and TPM-DMA with electron-donating groups, apart from electronic overlapping, a noticeable D-A transition from the donor to phenyl ring was observed. Together with their symmetrical structures and the shortened atomic distances of 1-2, 2-3 and 3-1 at $\mathrm{S}_{1}$ (Table S1), the dihedral angles between the reference plane and each phenyl ring decreased from $55^{\circ}$ to around $46^{\circ}$. This indicated the closer distance between every two phenyl rings and more concentrated $\pi$ electrons at the center of the molecular skeleton in comparison with $\mathrm{S}_{0}$. In TPM-CN, two phenyl rings displayed a nearly face-to-face conformation while the other one was perpendicular to them. Such a parallel arrangement was beneficial for efficient TSC, even though it was a binary rather than a ternary TSC, as proved in our previous report. ${ }^{29}$ The parallel binary TSC caused red-shift in clusteroluminescence from 402 to $466 \mathrm{~nm}$ (Figure 4d). For nitro-substituted TPM-NO ${ }_{2}$, a dark $\left(n, \pi^{*}\right)$ transition showing no intramolecular TSC became dominant, deciphering its nonfluorescent behavior in the solution and aggregate state. ${ }^{34}$ 


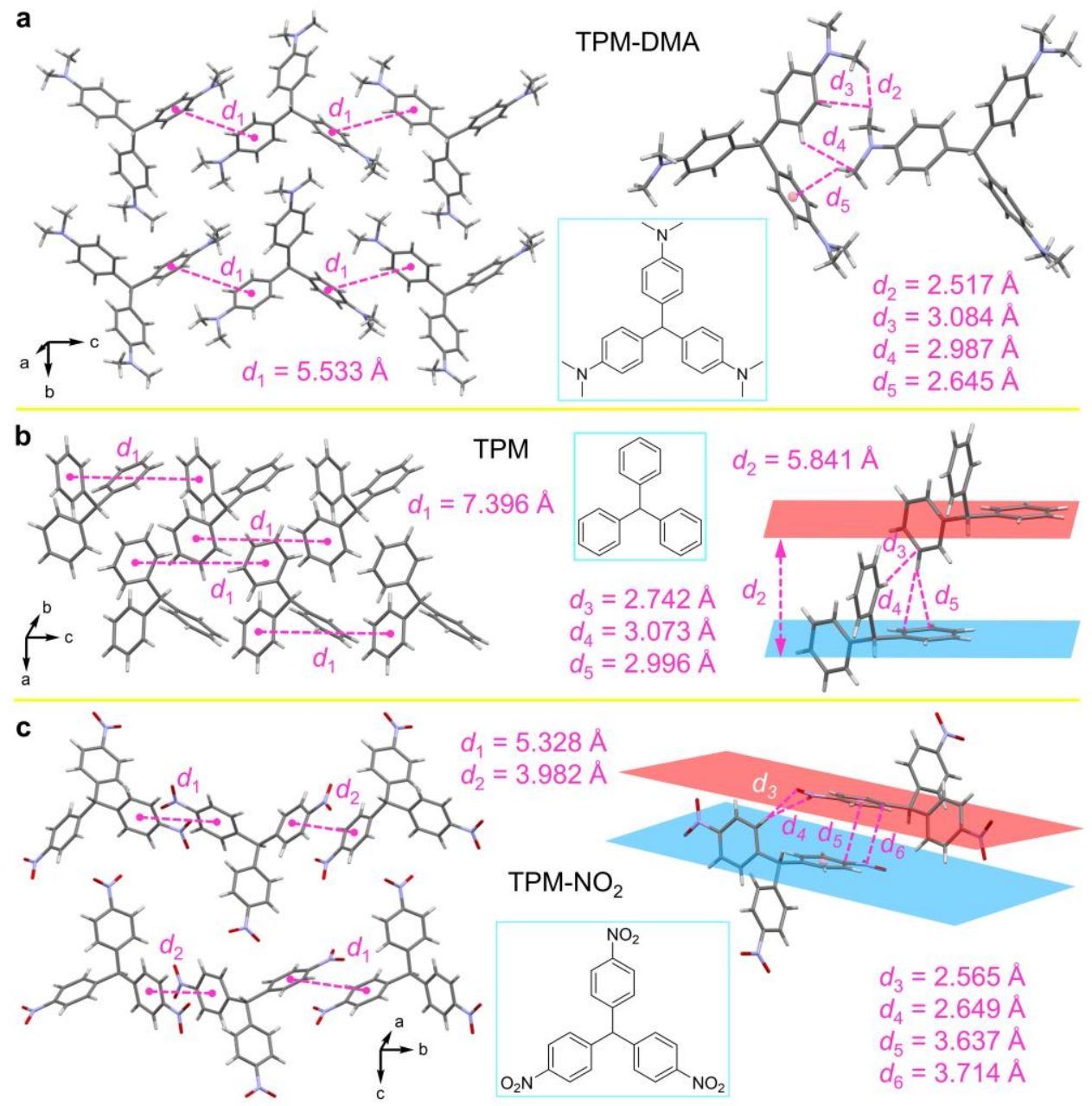

Figure 6. Crystal packing diagrams of (a) TPM-DMA, (b) TPM and (c) TPM-NO ${ }_{2}$

2.3 Crystal Structures. Single-molecule calculations had proved the important role of intramolecular TSC in clusteroluminescence of TPM derivatives, how about the intermolecular interaction? To explore the effect of intermolecular interaction on clusteroluminescence, crystal structures of all compounds were obtained and analyzed (Table S3). Figure 6 showed the crystal packing structures of TPM-DMA, TPM and TPM-NO 2 . In TPM-DMA, no parallel plane was observed and the centroid-centroid distance between two adjacent phenyl rings was $5.533 \AA$ (Figure 6a). Instead, some weak intermolecular interactions such as $\mathrm{C} \cdots \mathrm{H}, \mathrm{C}-\mathrm{H} \cdots \pi$ and $\mathrm{H} \cdots \mathrm{H}$ were dominant. A quantitative analysis on intermolecular interactions based on Hirshfeld surface was utilized. ${ }^{35}$ As showed in Figure 7, the full fingerprints appeared as grey shadows and the selected interaction was shown with blue. Thus, in TPM-DMA, the hydrogen-involved intermolecular interaction ( $\left.P_{\mathrm{HII}}\right)$ accounted for $99.7 \%$ of all intermolecular interactions. No intermolecular $\mathrm{C} \cdots \mathrm{C}$ interaction was detected, suggesting the absence of intermolecular TSC (Figure 7a and S18). The same situation was observed in TPM-MO, which showed almost no intermolecular TSC (Figure S19 and S20). In TPM, although the two intermolecular phenyl rings were parallel to each other, the distance between the two planes was as far as $5.841 \AA$ (Figure $6 \mathbf{b}$ ) and the $P_{\text {HII }}$ of TPM was up to $100 \%$ without intermolecular C...C interaction
(Figure $\mathbf{7 b}$ and $\mathbf{S 2 1}$ ). Therefore, the above results suggested that the clusteroluminescence of TPM and its donor-based derivatives was a kind of intramolecular rather than intermolecular behavior. Whereas these weak intermolecular interactions could restrict intramolecular motions and stabilize excitons with intramolecular TSC. ${ }^{30,36}$

For electron-withdrawing TPM-CN and TPM-NO $\mathrm{N}_{2}$, the closest intermolecular C...C distances determined from the crystal packing structures were 3.442 and $3.637 \AA$, respectively, which suggested strong intermolecular $\pi-\pi$ stacking (Figure 6c and S22). Meanwhile, intermolecular D-A interaction also existed, as proved by the presence of short intermolecular $\mathrm{C} \cdots \mathrm{N}$ distance. From the quantitative perspective, the proportion of intermolecular $\mathrm{C} \cdots \mathrm{C}$ interaction to total interactions $\left(P_{\mathrm{C} \cdots \mathrm{C}}\right)$ in TPM-CN and TPM$\mathrm{NO}_{2}$ was $8.4 \%$ and $6.3 \%$, respectively (Figure 7c, S23 and S24), which further confirmed the existence of intermolecular $\pi-\pi$ stacking. Therefore, the redder clusteroluminescence of electron-withdrawing TPM derivatives than TPM resulted from both intramolecular and intermolecular through-space interactions. Meanwhile, the close packing of TPM-CN and TPM- $\mathrm{NO}_{2}$ also brought the detrimental intermolecular photoinduced electron transfer from phenyl rings to cyano and nitro groups, which enhanced the nonradiative decay and decreased the efficiency of clusteroluminescence. 

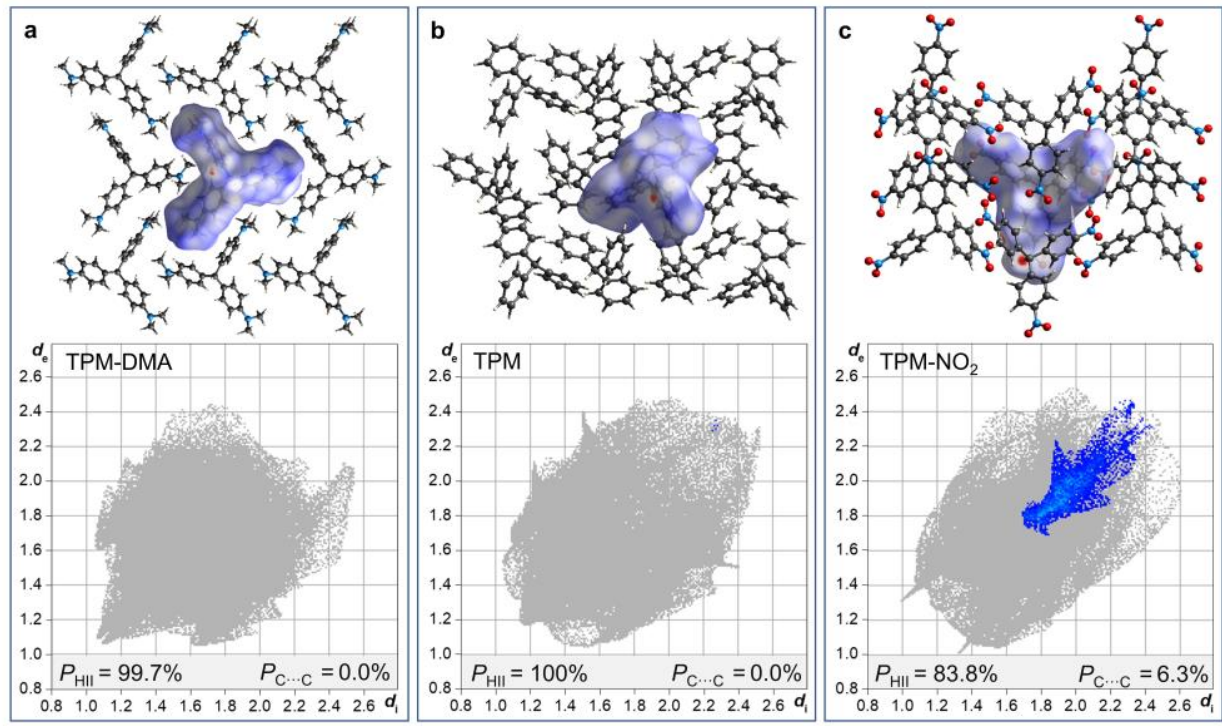

Figure 7. (Upper panel) Hirshfeld surfaces (mapped over $d_{\text {norm }}$ ) and (lower panel) decomposed fingerprint plots of (a) TPM-DMA, (b) TPM and (c) TPM-NO $\mathrm{N}_{2}$. The full fingerprints appeared as grey shadows underneath decomposed plots and intermolecular C...C interaction was shown as blue shadow. $P_{\mathrm{HII}}=$ proportion of hydrogen-involved intermolecular interaction to total intermolecular interaction, $P_{\mathrm{C} \cdots \mathrm{C}}=$ proportion of intermolecular $\mathrm{C} \cdots \mathrm{C}$ interaction to total intermolecular interaction.

$2.4 \quad$ Clusteroluminescence. Traditional clusteroluminogens based on macromolecular systems shows ambiguous spatial conformation and interaction, which makes it difficult to clarify the photophysical process of clusteroluminescence. In contrast, the identified singlemolecule and packing structures in the current system enable deep studies on the working mechanisms of clusterization-triggered emission. Reorganization energy $(\lambda)$ is a quantitative indicator of intrinsic geometry change upon photoexcitation, which also reflects the contribution of intramolecular motion to nonradiative decay. ${ }^{37-38}$ Figure 8a exhibited the $\lambda$ of TPM-DMA at different wavenumbers in the isolated phase as an example. The total $\lambda$ was 2142 $\mathrm{cm}^{-1}$ and $66.17 \%$ of it was contributed by the twisting motion of dihedral angle located mainly at low-frequency region $\left(<500 \mathrm{~cm}^{-1}\right)$. The low-frequency vibration modes also tended to mix upon excitation to activate multiple nonradiative decay pathways. Hence, the potential energy surface (PES) of TPM-DMA in dilute solution was proposed and displayed in Figure 8b. Upon photoexcitation to $S_{1}$, some excitons decayed via radiative channel to produce TBC emission. At the same time, transient species with intramolecular TSC also formed but immediately evolved to the dark state near the conical intersection due to the vigorous low-frequency motions..$^{39}$ As a result, only the TBC emission was detected. Based on the crystal packing structure of TPM-DMA, its aggregate environment was also simulated using ONIOM model with combined quantum mechanics and molecular mechanics (QM/MM) approach (Figure S25). The central molecule acted as the high layer with QM at B3LYP-D3/6-31G(d,p) level, while the surrounding molecules played as the low layer using universal force field (UFF). The total $\lambda$ in the aggregate state largely decreased to $1246 \mathrm{~cm}^{-1}$ and the contribution of dihedral angle also declined to $40.38 \%$ (Figure 8c), suggesting the restriction of intramolecular motion, especially twisting motion in the excited state. By combining experimental and theoretical results, Figure 8d drew the PES for TPM-DMA in the crystalline state. Similar to that in the solution state, some excited excitons released from the channel of TBC emission. However, according to Kasha's rule, more excitons went to the low-lying state of TSC which was further stabilized by restricted intramolecular motion. Thus, the nonradiative decay channel was blocked and clusteroluminescence was realized with comparatively high quantum yield.

Reorganization energy of other compounds in the isolated and crystalline phases was also calculated (Figure S26-S29). For TPM-MO and TPM, both total $\lambda$ and contribution of dihedral angle became smaller in the crystalline phase than those in the isolated phase. Besides, the increased $\Phi_{\text {TSC }}$ from TPM $(2.7 \%)$, then TPM-MO $(6.3 \%)$ and to TPM-DMA (6.9\%) was consistent with the enlarged $\Delta \lambda$ (the decline of $\lambda$ from isolated to crystalline phase: 33 $\mathrm{cm}^{-1}$ for TPM, $514 \mathrm{~cm}^{-1}$ for TPM-MO and $896 \mathrm{~cm}^{-1}$ for TPMDMA), verifying that the restriction of intramolecular motion in the crystalline state had stabilized the TSC and boosted the emission efficiency of clusteroluminescence. However, the total $\lambda$ of TPM-CN slightly increased from isolated $\left(2857 \mathrm{~cm}^{-1}\right)$ to crystalline phase $\left(3143 \mathrm{~cm}^{-1}\right)$ but the contribution of dihedral angle reduced from $57.34 \%$ to 26.95\% (Figure S28), which resulted in the decreased emission efficiency but reddest clusteroluminescence among all molecules. So, within this molecule, intramolecular motion performs like a double-edged sword that produces the low-lying TSC state but cannot stabilize the excited-state through-space interaction. TPM- $\mathrm{NO}_{2}$ is one of the extreme cases: its total $\lambda$ was large and measured to be 7316 and $7370 \mathrm{~cm}^{-1}$ in the isolated and crystalline phases, which quenched the emission in both phases (Figure S29). 

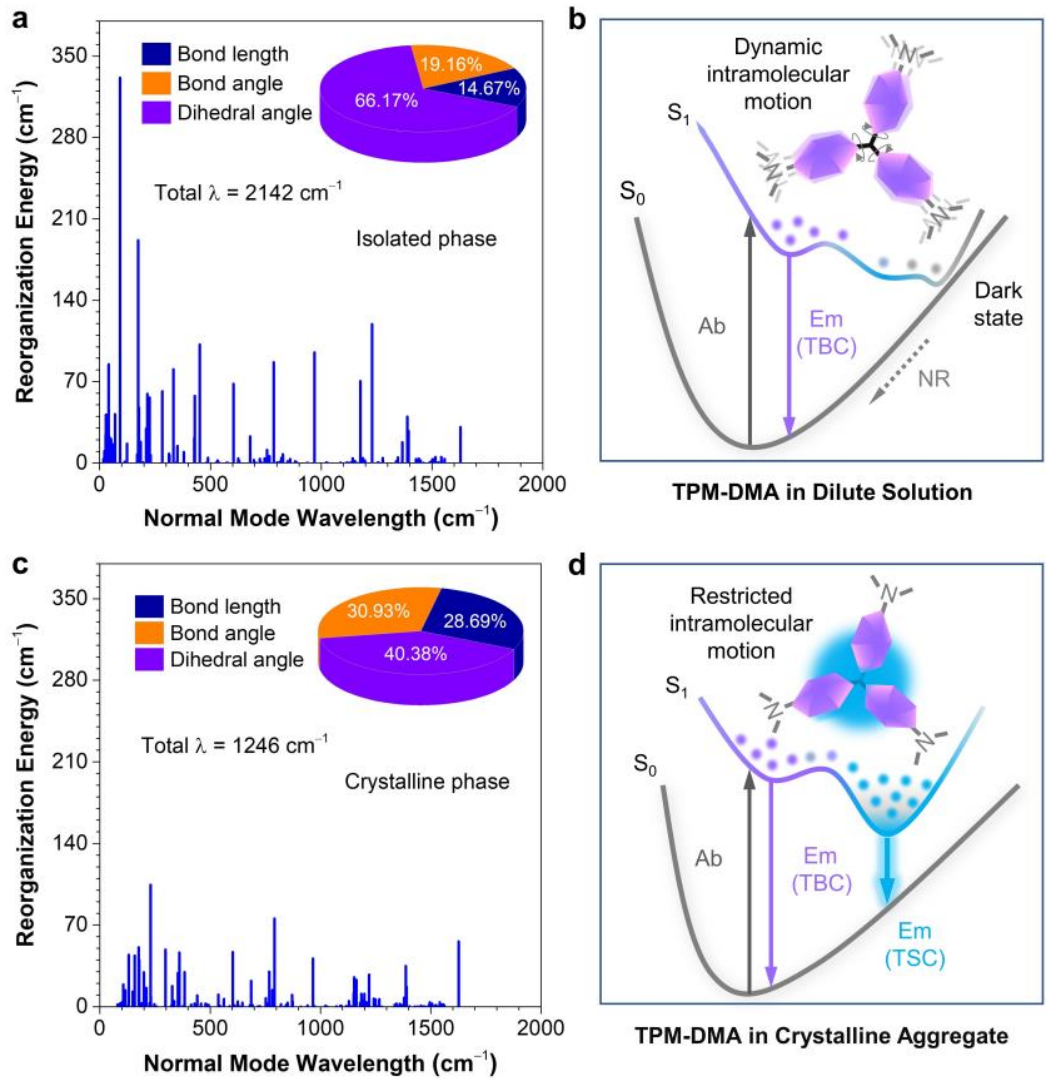

Figure 8. Plots of reorganization energy vs. normal mode wavenumber of TPM-DMA in (a) isolated and (c) crystalline phases calculated by TD-DFT, B3LYP-D3/6-31G(d,p), Gaussian 16 program. Inset: proportions of bond length, bond angle and dihedral angle contributed to total reorganization energy. Potential energy surfaces of TPM-DMA in (c) solution and (d) aggregate states. Ab $=$ absorption, $\mathrm{Em}=$ emission, and $\mathrm{NR}=$ nonradiative decay.

a

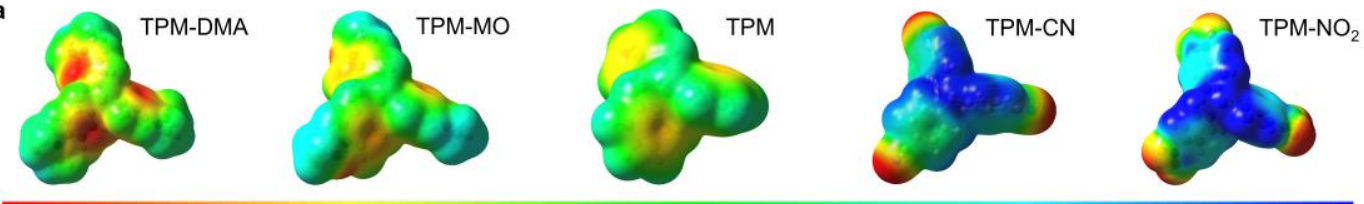

$-0.038$

b

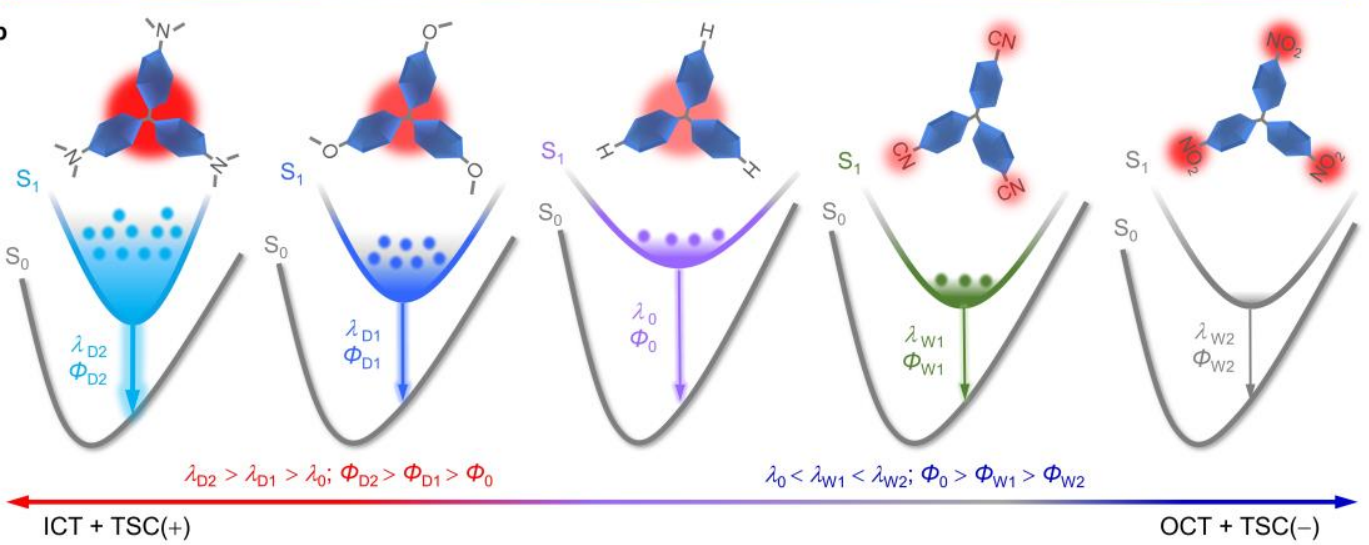

Figure 9. (a) Electronic static potential (ESP) mapped on the isosurface of electronic density, based on optimized excited-state geometries of TPM-R. Negative electrostatic potential (red) represented high electronic density, whereas positive one (blue) corresponded to low electronic density. The numbers of potential were given in au. (b) First line: effects of charge transfer (CT) and TSC; Second line: the proposed potential energy surfaces for the clusteroluminescence of TPM-R; ICT = inward charge transfer, OCT $=$ outward charge transfer,$+=$ stabilization/increment, $-=$ destabilization/decrement. 
To investigate how electron-donating or withdrawing groups affect TSC and clusteroluminescence, the excitedstate electronic static potential based on electronic density was mapped. As shown in Figure 9a, the red parts represented high electronic density, while those showing blue corresponded to low density. Apparently, electrondonating groups provided more electrons to the central part of molecular skeleton as the red color was observed on the three phenyl rings of TPM-DMA and TPM-MO. TPM, as the basic compound, showed lower electronic density at the central phenyl rings than its electron-donating derivatives. In contrast, TPM-CN and TPM-NO ${ }_{2}$ with strong electronwithdrawing groups exhibited low electronic density at the central phenyl rings, and the electrons were pulled to the exterior cyano and nitro groups. Accordingly, a complete picture of clusteroluminescence of these different systems was summarized from the perspective of intramolecular TSC (Figure 9b). In the excited state, electron-rich TPM derivatives pushed electrons from donors to the central phenyl rings, to trigger an inward charge transfer (ICT) process and stabilize the intramolecular TSC. Therefore, from TPM, then TPM-MO and finally to TPM-DMA, the wavelength and efficiency of clusteroluminescence became longer and higher. In contrast, TPM derivatives with electron-withdrawing groups attracted electrons from the central phenyl rings to the exterior acceptors, to produce an outward charge transfer (OCT) and destabilize the TSC. Finally, TPM-CN and TPM-NO $\mathrm{N}_{2}$ showed inefficient clusteroluminescence although the energy levels of their TSC states were lower than TPM and even their electronrich cousins.

\section{Conclusion}

In this work, nonconjugated triphenylmethane (TPM) and its derivatives with electron-donating (TPM-DMA and TPM-MO) and electron-withdrawing (TPM-CN and TPM$\mathrm{NO}_{2}$ ) groups were synthesized and their photophysical properties were systematically investigated. Except TPM$\mathrm{NO}_{2}$, all the molecules exhibited dual emission in the aggregate state: a short-wavelength peak from TBC and a long-wavelength clusteroluminescence stemmed from TSC. Meanwhile, introduction of the electron-donating groups had been found to red-shift the wavelength and increase the efficiency of the clusteroluminescence at the same time. Crystal packing and theoretical calculation suggested that the addition of donors not only increased the electronic density for TSC but also stabilized the formed excitons by restricting the intramolecular motion. However, the introduction of acceptors decreased the emission efficiency, but still caused a bathochromic shift in clusteroluminescence. Different from TPM and the electrondonating counterparts, TPM-CN and TPM- $\mathrm{NO}_{2}$ exhibited both intra- and intermolecular TSC to result in the reddest clusteroluminescence of TPM-CN. But the vigorous excitedstate intramolecular motion and unfavorable intermolecular PET process destabilized the excited excitons to eventually quench the light emission. This work not only proves the general mechanism of through-space conjugation for clusteroluminescence in nonconjugated luminogens but also provides a reliable strategy to manipulate its emission efficiency and color simultaneously. It is anticipated that the development of TSC theories will establish a significant linkage between molecular and aggregate photophysics.

\section{ASSOCIATED CONTENT}

Supporting Information. Materials and methods, synthetic procedures, characterization, crystallographic data (TPM-DMA, TPM-MO, TPM-CN and TPM-NO2) and computational details, including Figures S1-S29 and Tables S1-S13. This material is available free of charge via the Internet at http://pubs.acs.org

\section{AUTHOR INFORMATION}

\section{Corresponding Author}

*Haoke Zhang (zhanghaoke@zju.edu.cn);

*Ben Zhong Tang (tangbenz@cuhk.edu.cn)

\section{ORCID}

Jianyu Zhang: 0000-0002-5213-7063

Haoke Zhang: 0000-0001-7309-2506

Ben Zhong Tang: 0000-0002-0293-964X

\section{Notes}

The authors declare no competing financial interest.

\section{ACKNOWLEDGMENT}

This work was partially supported by the National Natural Science Foundation of China Grant (51773076, 21871060, 81271476 and 31870991), the Innovation and Technology Commission (ITC-CNERC14SC01), the Research Grants Council of Hong Kong (16305518, 16307020, C6014-20W, C6009-17G, and N-HKUST609/19), and the Material Science Foundation of Guangdong Province (2019B121205012 and 2019B030301003). H. Z thanks to the support from the Fundamental Research Funds for the Central Universities and the Open Fund of Guangdong Provincial Key Laboratory of Luminescence from Molecular Aggregates, and South China University of Technology (2019B030301003).

\section{REFERENCES}

(1) Al-Amri, M. D.; El-Gomati, M.; Zubairy, M. S., Optics in Our Time. Springer Nature: 2016.

(2) Wei, Q.; Fei, N.; Islam, A.; Lei, T.; Hong, L.; Peng, R.; Fan, X.; Chen, L.; Gao, P.; Ge, Z. Small-Molecule Emitters with High Quantum Efficiency: Mechanisms, Structures, and Applications in OLED Devices. Adv. Opt. Mater. 2018, 6, 1800512.

(3) Xu, W.; Wang, D.; Tang, B. Z. NIR-II AIEgens: A Win - Win Integration Towards Bioapplications. Angew. Chem. Int. Ed. 2021, 60, 7476-7487.

(4) Lin, T. A.; Chatterjee, T.; Tsai, W. L.; Lee, W. K.; Wu, M. J.; Jiao, M.; Pan, K. C.; Yi, C. L.; Chung, C. L.; Wong, K. T.; Wu, C. C. Sky-Blue Organic Light Emitting Diode with 37\% External Quantum Efficiency Using Thermally Activated Delayed Fluorescence from Spiroacridine-Triazine Hybrid. Adv. Mater. 2016, 28, 6976-6983.

(5) Zhang, D.; Duan, L.; Zhang, Y.; Cai, M.; Zhang, D.; Qiu, Y. Highly Efficient Hybrid Warm White Organic Light-Emitting Diodes Using a Blue Thermally Activated Delayed Fluorescence Emitter: Exploiting the External Heavy-Atom Effect. Light: Sci. Appl. 2015, 4, e232.

(6) Yamaguchi, Y.; Matsubara, Y.; Ochi, T.; Wakamiya, T.; Yoshida, Z. How the $\pi$ Conjugation Length Affects the Fluorescence Emission Efficiency. J. Am. Chem. Soc. 2008, 130, 13867-13869.

(7) An, B. K.; Gierschner, J.; Park, S. Y. $\pi$-Conjugated Cyanostilbene Derivatives: A Unique Self-Assembly Motif for Molecular Nanostructures with Enhanced Emission and Transport. Acc. Chem. Res. 2012, 45, 544-554. 
(8) Liu, S.; Zhang, H.; Li, Y.; Liu, J.; Du, L.; Chen, M.; Kwok, R. T. K.; Lam, J. W. Y.; Phillips, D. L.; Tang, B. Z. Strategies to Enhance the Photosensitization: Polymerization and the Donor-Acceptor EvenOdd Effect. Angew. Chem. Int. Ed. 2018, 57, 15189-15193.

(9) Zhao, J.; Wu, W.; Sun, J.; Guo, S. Triplet Photosensitizers: From Molecular Design to Applications. Chem. Soc. Rev. 2013, 42, 53235351.

(10) Shen, H.; Li, Y.; Li, Y. Self-Assembly and Tunable Optical Properties of Intramolecular Charge Transfer Molecules. Aggregate 2020, 1, 57-68.

(11) Xu, Z.; Wang, C.; Qiao, Q.; Chi, W.; Chen, J.; Liu, W.; Tan, D.; McKechnie, S.; Lyu, D.; Jiang, X.-F.; Zhou, W.; Xu, N.; Zhang, Q.; Liu, $X$. Quantitative Design of Bright Fluorophores and AIEgens via the Accurate Prediction of Twisted Intramolecular Charge Transfer (TICT). Angew. Chem. Int. Ed. 2020, 59, 10160-10172.

(12) Watson, W. F.; Livingston, R. Concentration Quenching of Fluorescence in Chlorophyll Solutions. Nature 1948, 162, 452-453. (13) Tu, Y.; Zhao, Z.; Lam, J. W. Y.; Tang, B. Z. Aggregate Science: Much to Explore in the Meso World. Matter 2021, 4, 338-349.

(14) Zhang, H.; Zhao, Z.; Turley, A. T.; Wang, L.; McGonigal, P. R.; Tu, Y.; Li, Y.; Wang, Z.; Kwok, R. T. K.; Lam, J. W. Y.; Tang, B. Z. Aggregate Science: From Structures to Properties. Adv. Mater. 2020, 32 2001457.

(15) Yang, J.; Fang, M.; Li, Z. Organic Luminescent Materials: The Concentration on Aggregates from Aggregation-Induced Emission. Aggregate 2020, 1, 6-18.

(16) Luo, J.; Xie, Z.; Lam, J. W.; Cheng, L.; Chen, H.; Qiu, C.; Kwok, H. S.; Zhan, X.; Liu, Y.; Zhu, D.; Tang, B. Z. Aggregation-Induced Emission of 1-Methyl-1,2,3,4,5-Pentaphenylsilole. Chem. Commun. 2001, 1740-1741.

(17) Zhao, Z.; Zhang, H.; Lam, J. W. Y.; Tang, B. Z. AggregationInduced Emission: New Vistas at Aggregate Level. Angew. Chem. Int Ed. 2020, 59, 9888-9907.

(18) Mei, J.; Leung, N. L.; Kwok, R. T.; Lam, J. W.; Tang, B. Z. Aggregation-Induced Emission: Together We Shine, United We Soar! Chem. Rev. 2015, 115, 11718-11940.

(19) Yanari, S. S.; Bovey, F. A.; Lumry, R. Fluorescence of Styrene Homopolymers and Copolymers. Nature 1963, 200, 242-244.

(20) Ye, R.; Liu, Y.; Zhang, H.; Su, H.; Zhang, Y.; Xu, L.; Hu, R.; Kwok, R. T. K.; Wong, K. S.; Lam, J. W. Y.; Goddard, W. A.; Tang, B. Z. NonConventional Fluorescent Biogenic and Synthetic Polymers without Aromatic Rings. Polym. Chem. 2017, 8, 1722-1727.

(21) He, B.; Zhang, J.; Zhang, J.; Zhang, H.; Wu, X.; Chen, X.; Kei, K. H. S.; Qin, A.; Sung, H. H. Y.; Lam, J. W. Y.; Tang, B. Z. Clusteroluminescence from Cluster Excitons in Small Heterocyclics Free of Aromatic Rings. Adv. Sci. 2021, 2004299.

(22) Braendle, A.; Perevedentsev, A.; Cheetham, N. J.; Stavrinou, P. N.; Schachner, J. A.; Mösch-Zanetti, N. C.; Niederberger, M.; Caseri, W. R. Homoconjugation in Poly(phenylene methylene)s: A Case Study of Non- $\pi$-Conjugated Polymers with Unexpected Fluorescent Properties. J. Polym. Sci., Part B: Polym. Phys. 2017, 55, 707-720.

(23) Zhou, Q.; Yang, T.; Zhong, Z.; Kausar, F.; Wang, Z.; Zhang, Y.; Yuan, W. Z. Clustering-Triggered Emission Strategy Towards Tunable Multi-Color Persistent Phosphorescence. Chem. Sci. 2020, 11, 2926-2933.

(24) Zhang, H.; Zhao, Z.; McGonigal, P. R.; Ye, R.; Liu, S.; Lam, J. W. Y.; Kwok, R. T. K.; Yuan, W. Z.; Xie, J.; Rogach, A. L.; Tang, B. Z.
Clusterization-Triggered Emission: Uncommon Luminescence from Common Materials. Mater. Today 2019, 32, 275-292.

(25) Wang, Z.; Zhang, H.; Li, S.; Lei, D.; Tang, B. Z.; Ye, R. Recent Advances in Clusteroluminescence. Top. Curr. Chem. 2021, 379, 14.

(26) Zhang, X.; Du, L.; Zhao, W.; Zhao, Z.; Xiong, Y.; He, X.; Gao, P. F.; Alam, P.; Wang, C.; Li, Z.; Leng, J.; Liu, J.; Zhou, C.; Lam, J. W. Y.; Phillips, D. L.; Zhang, G.; Tang, B. Z. Ultralong UV/Mechano-Excited Room Temperature Phosphorescence from Purely Organic Cluster Excitons. Nat. Commun. 2019, 10, 5161.

(27) Zhao, Z.; Wang, Z.; Tavakoli, J.; Shan, G.; Zhang, J.; Peng, C.; Xiong, Y.; Zhang, X.; Cheung, T. S.; Tang, Y.; Huang, B.; Yu, Z.; Lam, J. W. Y.; Tang, B. Z. Revisiting an Ancient Inorganic AggregationInduced Emission System: An Enlightenment to Clusteroluminescence. Aggregate 2021. DOI: 10.1002/agt2.36

(28) Shao, S.; Wang, L. Through-Space Charge Transfer Polymers for Solution-Processed Organic Light-Emitting Diodes. Aggregate 2020, $1,45-56$.

(29) Zhang, H.; Zheng, X.; Xie, N.; He, Z.; Liu, J.; Leung, N. L. C.; Niu, Y.; Huang, X.; Wong, K. S.; Kwok, R. T. K.; Sung, H. H. Y.; Williams, I. D.; Qin, A.; Lam, J. W. Y.; Tang, B. Z. Why Do Simple Molecules with "Isolated" Phenyl Rings Emit Visible Light? J. Am. Chem. Soc. 2017, 139, 16264-16272.

(30) Zhang, J.; Zhang, H.; Lam, J. W. Y.; Tang, B. Z. Restriction of Intramolecular Motion (RIM): Investigating AIE Mechanism from Experimental and Theoretical Studies. Chem. Res. Chin. Univ. 2021, 37, 1-15.

(31) Tu, Y.; Yu, Y.; Xiao, D.; Liu, J.; Zhao, Z.; Liu, Z.; Lam, J. W. Y.; Tang, B. Z. An Intelligent Aiegen with Nonmonotonic Multiresponses to Multistimuli. Adv. Sci. 2020, 7, 2001845.

(32) Mu, Y.; Liu, Y.; Tian, H.; Ou, D.; Gong, L.; Zhao, J.; Zhang, Y.; Huo, Y.; Yang, Z.; Chi, Z. Sensitive and Repeatable Photoinduced Luminescent Radicals from a Simple Organic Crystal. Angew. Chem. Int. Ed. 2020, 60, 6367-6371.

(33) Ai, X.; Evans, E. W.; Dong, S.; Gillett, A. J.; Guo, H.; Chen, Y.; Hele, T. J. H.; Friend, R. H.; Li, F. Efficient Radical-Based Light-Emitting Diodes with Doublet Emission. Nature 2018, 563, 536-540.

(34) Zhao, W.; He, Z.; Peng, Q.; Lam, J. W. Y.; Ma, H.; Qiu, Z.; Chen, Y.; Zhao, Z.; Shuai, Z.; Dong, Y.; Tang, B. Z. Highly Sensitive Switching of Solid-State Luminescence by Controlling Intersystem Crossing. Nat. Commun. 2018, 9, 3044.

(35) Spackman, M. A.; Jayatilaka, D. Hirshfeld Surface Analysis. CrystEngComm 2009, 11, 19-32.

(36) Leung, N. L.; Xie, N.; Yuan, W.; Liu, Y.; Wu, Q.; Peng, Q.; Miao, Q.; Lam, J. W.; Tang, B. Z. Restriction of Intramolecular Motions: The General Mechanism Behind Aggregation-Induced Emission. Chem. Eur. J. 2014, 20, 15349-15353.

(37) Zhang, H.; Liu, J.; Du, L.; Ma, C.; Leung, N. L. C.; Niu, Y.; Qin, A.; Sun, J.; Peng, Q.; Sung, H. H. Y.; Williams, I. D.; Kwok, R. T. K.; Lam, J. W. Y.; Wong, K. S.; Phillips, D. L.; Tang, B. Z. Drawing a Clear Mechanistic Picture for the Aggregation-Induced Emission Process Mater. Chem. Front. 2019, 3, 1143-1150.

(38) Shuai, Z.; Peng, Q. Organic Light-Emitting Diodes: Theoretical Understanding of Highly Efficient Materials and Development of Computational Methodology. Natl. Sci. Rev. 2017, 4, 224-239.

(39) Tu, Y.; Liu, J.; Zhang, H.; Peng, Q.; Lam, J. W. Y.; Tang, B. Z. Restriction of Access to the Dark State: A New Mechanistic Model for Heteroatom-Containing Aie Systems. Angew. Chem. Int. Ed. 2019, 58, 14911-14914. 


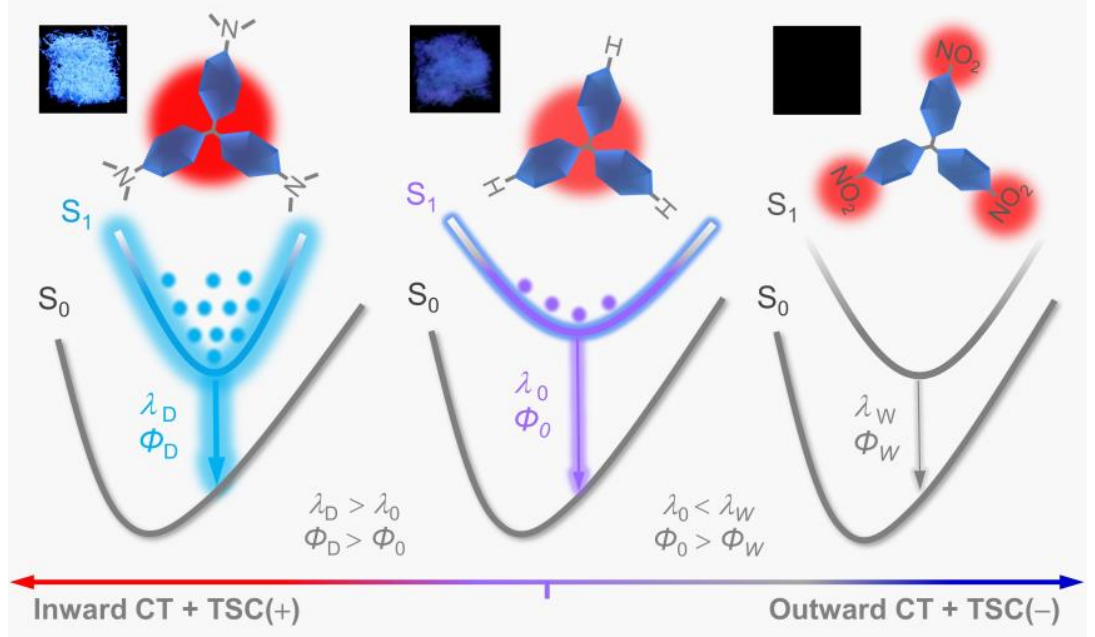

